\title{
Correction to "Approach to Leg Edema of Unclear Etiology"
}

In the abovementioned article (Ely JW, Osheroff JA, Chambliss ML, Ebell MH. J Am Board Fam Med. 2006;19(2):148-160), the following sentence was incorrect in the abstract. The sentence should read as follows: Patients who have findings consistent with sleep apnea, such as daytime somnolence, loud snoring, or neck circumference $>17$ inches, should be evaluated for pulmonary hypertension with an echocardiogram. We apologize for the error, and we regret any confusion or inconvenience it may have caused. 